\title{
Ground-based measurements of ionospheric dynamics
}

\author{
Daniel Kouba* and Jaroslav Chum \\ Institute of Atmospheric Physics, Czech Academy of Sciences, Prague, Czech Republic
}

Received 13 June 2017 / Accepted 20 March 2018

\begin{abstract}
Different methods are used to research and monitor the ionospheric dynamics using ground measurements: Digisonde Drift Measurements (DDM) and Continuous Doppler Sounding (CDS). For the first time, we present comparison between both methods on specific examples. Both methods provide information about the vertical drift velocity component. The DDM provides more information about the drift velocity vector and detected reflection points. However, the method is limited by the relatively low time resolution. In contrast, the strength of CDS is its high time resolution. The discussed methods can be used for real-time monitoring of medium scale travelling ionospheric disturbances. We conclude that it is advantageous to use both methods simultaneously if possible. The CDS is then applied for the disturbance detection and analysis, and the DDM is applied for the reflection height control.
\end{abstract}

Keywords: Ionosphere / remote sensing / vertical plasma drift / digisonde / continuous doppler sounding

\section{Introduction}

Sounding of the ionosphere using ionosondes is an important input for real-time monitoring and forecasting the state of the ionosphere and space weather impacts. The vertical ionospheric sounding is the traditional method for obtaining information about the profile of electron concentration. It was first applied in the early days of ionospheric research and is still used today. In addition, total electron content (TEC) started to be measured widely by global navigation satellite system (GNSS) receivers in recent decades. Their dense network allows application of tomographic methods to obtain not only the TEC maps but also 3D distribution of electron density on the regional or global scales and its temporal evolution (Hajj et al., 1994; Saito et al., 1998; Jakowski et al., 2005). The incoherent scatter radars are also used to provide electron densities, temperatures and plasma drift velocities (Banks et al., 1974; Fejer et al., 1991). However, low amount of them around the world is a limitation for global studies.

A relatively dense distribution of modern ionosondes in some areas primarily provides a network of classical ionogram sounding. Some modern digital ionosondes, especially Digisondes, are able to perform additional special measurements, e.g. drift measurements which can be routinely performed (Reinisch et al., 1998). There are tens of Digisondes worldwide which routinely measure drifts using Digisonde Drift Measurements (DDM) method. This technique has been validated by comparison with incoherent scatter radar

\footnotetext{
*Corresponding author: kouba@ufa.cas.cz
}

measurements at Sondrestrom and Jicamarca (Scali et al., 1995; Bertoni et al., 2006).

The Continuous Doppler Sounding (CDS) has been used to study ionospheric fluctuations on short time scales, including the periods of infrasound, since the late 1950s and early 1960s (Davies et al., 1962; Davies and Baker, 1966; Georges, 1973). As it is continuous sounding, it is especially suitable for investigation of wave processes. The CDS does not provide an information about electron density profile.

DDM and CDS can be used for identification of travelling ionospheric disturbances (TIDs) or other ionospheric disturbances in real-time. Kouba and Koucká Knížová (2016) describe behavior of the vertical drift component for quiet conditions. The Digisonde automatically calculates drift velocity vector after each drift measurements. Significant deviations from the quiet behavior can be automatically evaluated as a disturbance and the alert can be published immediately. Moreover, the CDS allows to obtain the basic TID characteristics. The speed and direction of TIDs caused by atmospheric gravity waves can be estimated from comparison of the signals recorded on different sounding paths (Chum et al., 2014). TIDs were also studied from TEC perturbations caused by acoustic gravity waves by using dense networks of dual-frequency GPS (GNSS) receivers (Nishioka et al., 2013; Otsuka et al., 2013; Lay et al., 2015). Fišer et al. (2017), based on comparison of the result obtained by CDS with the result obtained by Chou et al. (2017) from GPS-TEC measurements, showed that the multi-point CDS and a dense network of GPS-TEC measurements often provide similar propagation velocities and directions of GWs for distinct perturbations. 
All the ionospheric measurements are sensitive to ionospheric irregularities, e.g. spread F. Spread F, especially at low latitudes, can significantly deteriorate the quality of GPS (GNSS) signal and decrease the precision of position determination. Chum et al. (2016b) compared the observation of equatorial (low-latitude) spread $\mathrm{F}$ by ionosondes, CDSs, and GPS (GNSS) receivers. The spread F was detected by GPS receivers as high values of the scintillation index $\mathrm{S} 4$. Both the ground based measurements and satellite radio occultation measurements of S4 were used. Chum et al. (2016b) studied occurrence rates of spread $\mathrm{F}$, longitudinal distribution of spread $\mathrm{F}$ occurrence rates and zonal drift velocities of spread $\mathrm{F}$ and concluded that all the instruments provided consistent results.

In this paper, we provide a detailed comparison of DDM measurements with CDS. Section 2 describes and compares both measurement techniques in detail. Section 3 provides examples of measurements performed under different ionospheric conditions. The examples for both the quiet and disturbed days are given. Section 4 concludes with a brief summary.

\section{Instruments and techniques}

\subsection{Digisonde drift measurements (DDM)}

The drift measurements are typically performed shortly after ionogram sounding. Autoscaling process determines basic characteristics of the ionogram which are used for the following drift measurements. Digisonde selects sounding frequencies for the drift measurements according to the critical frequency foF 2 of the F layer (autodrift mode), or other chosen parameters (e.g., $f o E$ ). A receiving antenna array allows detecting locations of reflection points in the ionosphere for the signals transmitted during the measurements. The graphi graphical representation of such result is a SKYmap, which shows locations of the reflection points in the east-west and north-south plane. The values of Doppler shift are distinguished by the color of the points. The Doppler frequency shifts $D i$ for individual echoes are proportional to the line-ofsight velocity $(\boldsymbol{k} i \cdot \boldsymbol{v}): D i=-2 f_{0} / c(\boldsymbol{k} i \cdot \boldsymbol{v})$, where $f_{0}$ is the sounding frequency, $c$ is the speed of light and $k i$ is directional unit vector of $i$-th detected reflection point.

The drift velocity vector is estimated from the locations of detected reflection points and their Doppler frequency shifts. Digisondes provide real-time drift velocity estimation automatically using all detected reflection points. However, for more precise drift velocity analysis it is necessary to select carefully the reflection points manually (Kouba et al., 2008). The final products of DDM are three drift velocity components, i.e. vertical $\left(v_{z}\right)$, northward $\left(v_{N}\right)$, and eastward $\left(v_{E}\right)$ components, or alternatively vertical and horizontal velocity components and azimuth.

In practice, a fair amount of reflection points is detected during the measurements. The quantity varies usually between tens and thousands in dependence on current conditions. Therefore, the estimation of drift velocity vector components is obtained from overdetermined linear system of equations (Tarantola, 2005). The standard deviations for individual components are calculated using statistical methods. Typically, there are large differences in the accuracy of estimated vertical and horizontal components. While the vertical component of the drift velocity can be determined in most cases, there are often gaps in the time series of horizontal components, and their quality is significantly varying. Therefore, Kouba and Koucká Knížová (2016) studied only vertical component in quiet conditions. Systematic study of the horizontal components is more difficult and has not yet been performed. This study is therefore based on the vertical component of drift velocity obtained from the DDM.

\subsection{DDM in Pruhonice}

A Digisonde DPS4D installed at the Pruhonice observatory (N50, E14.5) provides routine drift measurements for both E and $\mathrm{F}$ regions. Digisonde in Pruhonice typically measures ionograms with 15 minutes' cadence. Ionogram measurements are followed by F-region drift measurements in autodrift mode and E-region drift measurements with fixed soundingfrequency window.

Special campaigns with a higher time resolution were also carried out additionally to the standard settings (e.g. the special campaign presented in this paper in Sect. 3.1).

\subsection{Continuous doppler sounding (CDS)}

The Doppler system used in this study was developed at the Institute of Atmospheric Physics, Prague. It is composed of several transmitters distributed in the western part of the Czech Republic and one receiver located in Prague. Currently the Doppler system operates at frequencies of $3.59 \mathrm{MHz}$, 4.65 MHz, and 7.0 MHz. To compare with DDM, we use signal from transmitter $\mathrm{Tx} 2$ which is collocated with the Digisonde and recorded by receiver located at about $7 \mathrm{~km}$ distance.

In the latter cases, the Doppler shift cannot be simply related to the movement of reflecting layer; e.g., if the air (plasma) fluctuations are caused by infrasound waves, then the compression or rarefaction of the gas can contribute to the observed Doppler shift more than the advection (up and down motion of the reflecting layer).

A short description of CDS and review of recent research applications of this system, including similar CDSs in other countries, was given by Laštovička and Chum (2017).

\subsection{DDM and CDS properties}

Each receiving antenna in the antenna array of the DPS4D receives a signal and its spectrum is subsequently analysed during DDM. The direction of the signal arrival is calculated for detected peaks in spectra and the individual source points (reflection points in the ionosphere) are identified. A set of these points can be displayed in the SKYmap. Finally, the unique velocity vector of the ionospheric plasma at selected height range is estimated for detected source points by means of Least-Squares fitting (Kouba et al., 2008).

The CDS measures a spectrum of a signal for each transmitter-receiver pair. The temporal evolution of the spectrum with time is usually presented in the form of a spectrogram. Several spatially separated transmitter-receiver (sounding) paths are usually used to determine horizontal velocities of ionospheric disturbances from the time delays 
Table 1. CDS vs. DDM: comparison of basic parameters.

\begin{tabular}{|c|c|c|}
\hline & CDS & DDM \\
\hline Time resolution & $\sim 10 \mathrm{~s}$ & Typically 15 or 5 min \\
\hline Spatial information & $\begin{array}{l}\text { Reflection points are assumed in midpoints } \\
\text { between } T x \text { and } \mathrm{Rx}\end{array}$ & Location of reflection points is displayed in SKYmaps \\
\hline Virtual height resolution & None. Reflection heights are estimated from ionograms & Typically $5 \mathrm{~km}$ \\
\hline Measurement duration & Continuous & $\sim 1 \mathrm{~min}$ \\
\hline Sounding frequency & Fixed & Fixed or related to critical frequencies, programmable \\
\hline
\end{tabular}

between the observations of corresponding disturbances on different sounding paths (Chum et al., 2014).

It is necessary to note that the velocity of the disturbance mentioned here is generally different from the horizontal component of the drift velocity vector obtained by the DDM. The DDM detects the electron motion and CDS detects movement of the structure/disturbance. In this study, spectrograms for one trace are used for comparison with the DDM results.

The sounding path between transmitter $\mathrm{Tx} 2$, which is located at about $1 \mathrm{~km}$ distance from DPS4D in Pruhonice, and receiver $\mathrm{Rx}$ located in Prague, about $7 \mathrm{~km}$ from Tx2, can be considered as vertical sounding. So, both Tx2-Rx path of CDS and DPS4D detect the signal reflected from the same part of the ionosphere, provided that they transmit at the same frequency.

DDM and CDS use different types of signals. The CDS transmits a harmonic wave and analyses the spectrum of the received signal. The DDM transmits coded pulses and detects peaks in the spectrum of the received signal. These peaks and their amplitudes are linked with the individual reflection points. Therefore, it is not a priori expectable that we would obtain similar characteristics for DDM and CDS (peak width etc.).

Table 1 shows a basic comparison of several parameters and measurement capabilities of both methods.

The advantage of CDS is its relatively high temporal resolution of about $10 \mathrm{~s}$, which is a compromise between high time resolution and sufficient frequency resolution. The spectrum is usually calculated over overlapping time intervals to get smooth spectrograms. The width of spectral intensity peaks in the Doppler shift spectrogram directly provides information about the uncertainty in the Doppler shift of the received signal.

The main Digisonde measurement is the vertical ionospheric sounding (ionogram measurements) and the measurement of an individual ionogram takes typically few minutes. One drift measurements takes about one minute, depending on the particular setting. The Digisonde typically performs routine drift measurements every $15 \mathrm{~min}$. Several stations use a higher cadence of 5 minutes. The higher cadence of measurements is also used during special short-term campaigns, e.g. when studying the solar eclipse effects. As the DDM runs in simultaneous mode with the standard vertical sounding according to the schedule, it is impossible to significantly improve its time resolution.

The advantage of DDM compared to CDS is the possibility of choosing/changing the sounding frequency. The DDM can be triggered in one of two basic modes: first, the sounding frequency (sounding frequency window) is fixed for all drift measurements; second, the sounding frequency depends on the critical frequency determined from the preceding ionogram (autodrift mode). The latter mode allows optimizing the sounding frequency according to the current conditions. Generally, different sounding frequency is used for each measurement in the autodrift mode.

The CDS uses a fixed frequency that cannot be changed. This can complicate a long-time measurements due to the temporal evolution of the ionospheric parameters, e.g., critical frequency foF2 can became lower than the sounding frequency, which results in loss of the signal. A possible solution of this problem is a simultaneous measurement at several sounding frequencies.

Spectrograms for different sounding frequencies can show behavior in different ionospheric layers (e.g. E, Es, F). For the correct interpretation of the spectrogram it is necessary to know which layer reflected the signal. The height at which the CDS sounding frequency was reflected can be determined from an ionogram measured by a nearby ionosonde. Therefore, the collocation of ionosonde is appropriate for a proper CDS interpretation.

In addition, drift measurements provide SKYmaps that show the spatial distribution of the reflection points. In the case of CDS spectrogram, there is no information about the spatial distribution of the reflection points.

\subsection{Comparison of DDM and CDS results}

In order to compare the results of both methods, a comparative characteristic must be chosen appropriately. Although both methods are based on Doppler shift phenomena, the value of the measured Doppler shift is not appropriate characteristic to compare their results. The Doppler shift is proportional to the sounding frequency and different sounding frequencies can be used for DDM and CDS. The direct comparison of the detected Doppler shift is possible only if the measurements are performed with the same sounding frequency. The DDM routinely uses the autodrift mode for $F$ layer measurement and the sounding frequency thus varies in dependence on the foF 2 change. Moreover, the DDM usually does not use a single sounding frequency but a set of frequencies (frequency window). In order to compare both methods directly, we performed a special campaign that will be discussed in Section 3.1.

Generally, the easiest way to compare DDM and CDS is to recalculate the detected Doppler shift values $D_{f}^{i}$ for $i$-th reflection point to its line-of-sight velocity $v_{\text {los }}^{i}$.

$$
v_{l o s}^{i}=-c D_{f}^{i} /\left(2 f_{0}^{i}\right)
$$




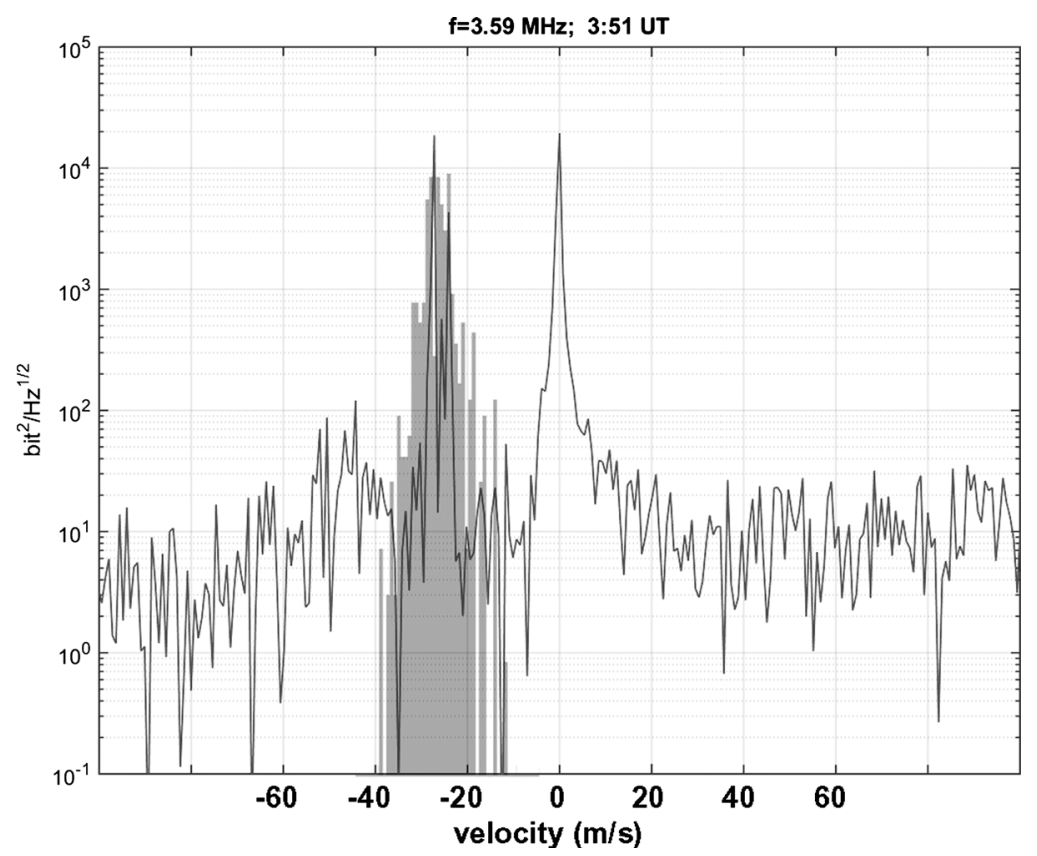

Fig. 1. Comparison of detected $v_{\text {los }}$ histogram for DDM on Sep 21 2016, at 03:51 UT (filled bars) and the CDS spectra for the same time (thin line). Both methods detect the peak in the same position. The additional strong peak at zero value in the CDS spectra is caused by ground wave.

The histogram of $v_{l o s}$ values for all detected reflection points can be drawn in the case of DDM.

The sounding frequency is fixed in the case of CDS. The Doppler shift of $1 \mathrm{~Hz}$ corresponds to the line-of-sight velocity of $-41.8 \mathrm{~m} / \mathrm{s}$ for sounding frequency of $3.59 \mathrm{MHz}$. For sounding frequency of $4.65 \mathrm{MHz}, 1 \mathrm{~Hz}$ corresponds to the velocity of $-32.3 \mathrm{~m} / \mathrm{s}$.

The example of the CDS spectra and histogram of detected line-of-sight velocities on SKYmap for measurements on August 13, 2012 at 03:51 is shown in Figure 1. The CDS spectrum for sounding frequency $3.59 \mathrm{MHz}$ was calculated for one minute interval at 03:51 UT. In the same time the DDM was performed with sounding frequencies of $2.7-3.05 \mathrm{MHz}$. The duration of DDM was $46 \mathrm{~s} .362$ reflection points were detected during the measurements. The histogram of line-ofsight velocities detected by DDM is presented with filled bars in Figure 1. The thin line represents CDS spectrum. Both methods provide a pronounced peak between about -20 to $-30 \mathrm{~m} / \mathrm{s}$.

The CDS spectra contains another strong narrow peak around zero value. Ground wave on a short path between transmitter Tx2 in Pruhonice and receiver Rx in Prague (distance about $7 \mathrm{~km}$ ) is responsible for this peak.

A similar peak is not observed in DDM. The Digisonde disconnects the receiving antennas for about $0.5 \mathrm{~ms}$ after transmitting the measuring pulse. Therefore, the first detected signal can be reflected by the ionospheric E layer and direct (ground) wave is not detected because arrived earlier. Moreover, only the signal corresponding to the reflection heights between 150 and $600 \mathrm{~km}$ is processed for $\mathrm{F}$ region DDM.

We compared the CDS spectra and DDM histograms for various cases - different DDM settings, significantly different DDM sounding frequency windows in comparison with CDS sounding frequencies, different seasons and daytime, etc. For the same CDS and DDM sounding frequencies reflected from the same ionospheric region (E, Es, F1, or F2) we get similar characteristic for both methods. The complication of CDS is that the sounding reflection sometimes switches between different layers ( $F-E s, F 1-F 2$, etc.).

We can also analyze the temporal evolution of the spectrum using CDS. It is appropriate to use spectrograms instead single spectral curves to explore it. We can add the result of DDM to the spectrogram. The above-mentioned histograms are shown in the spectrogram with the so called "colored bars representation" which are the vertical lines at the times of DDM measurements. This comparison will be used below. The spectral width of the received CDS signal can vary. If the wave reflects from a specially limited region that experiences the same movement, the peak of spectral intensity is relatively sharp, its width being similar as for the ground wave (Fig. 1). However, if the sounding wave undergoes multiple reflection from different regions as happens e.g. in the case of spread $\mathrm{F}$ conditions, then there is no sharp peak of spectral intensity; the peak can be relatively flat and exhibit many local maxima resembling noise. The received spectral intensity is colorcoded in the Doppler shift spectrograms. The colors thus provide direct information about the uncertainty of the Doppler shift (spectral width) of the received signal. The uncertainty also depends on the length of the time interval over which the spectrum is calculated.

\section{Data}

\subsection{Direct comparison of DDM and CDS measurements}

A special campaign was performed for detailed comparison of both methods on September 22, 2016. The Digisonde performed vertical ionospheric sounding (ionograms) every 


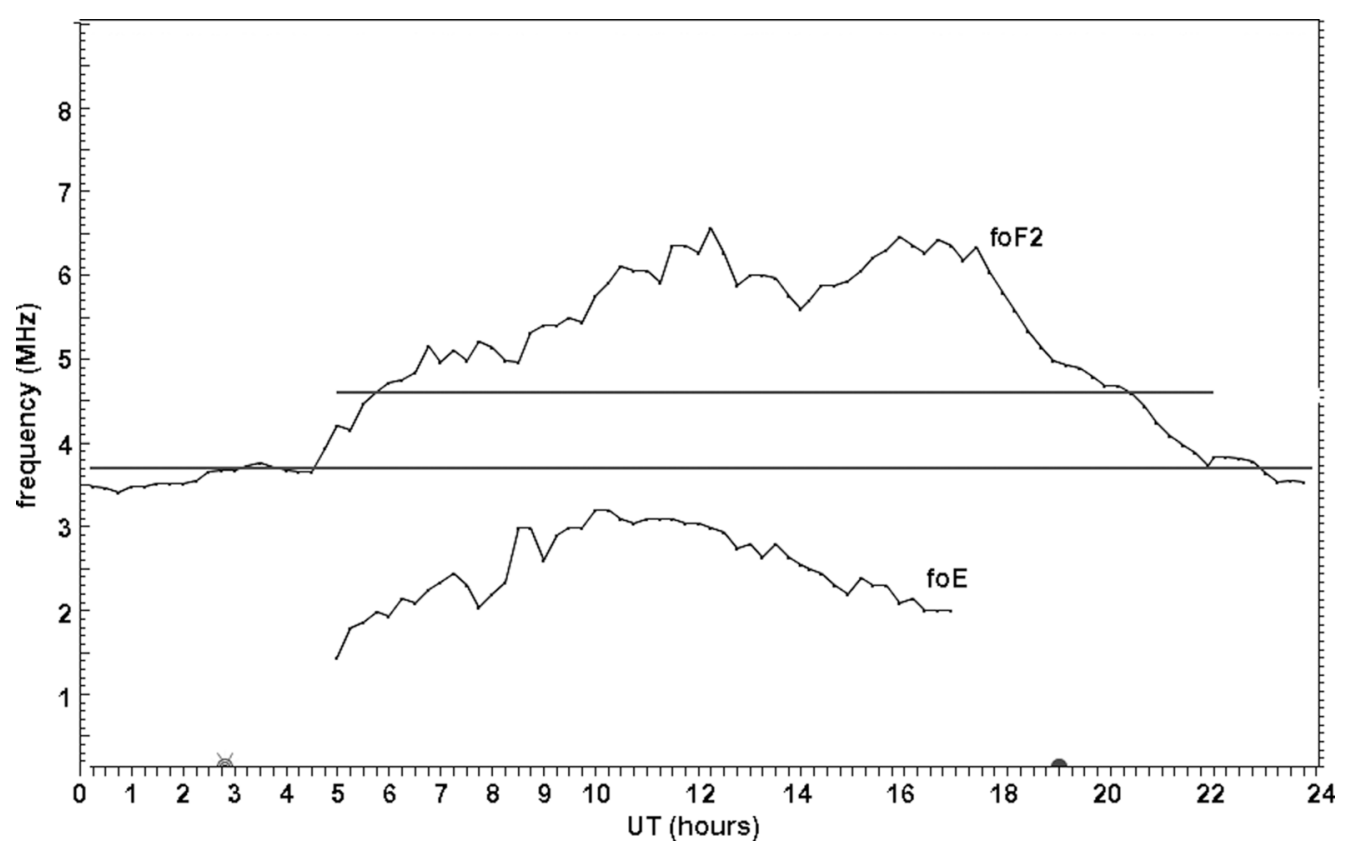

Fig. 2. The one-day plot of critical frequencies $f o E$ and $f o F 2$ measured at Pruhonice during the campaign on September 22, 2016. Two sounding frequencies used for the drift measurement and CDS are shown as a horizontal lines.

$15 \mathrm{~min}$. The whole working time between ionogram measurements was dedicated to the sequence of drift measurements during the campaign.

In the day-time schedule (05-22 UT) the drift measurements were performed at fixed frequencies corresponding to the frequencies of Doppler transmitters working at 3.59 and 4.65 MHz (DDM frequencies were slightly shifted to 3.58 and $4.655 \mathrm{MHz}$ to avoid a possible interference). Only the sounding frequency of $3.58 \mathrm{MHz}$ was used at night (2205 UT). Digisonde was able to perform 8 drift measurements after each ionog ram in $15 \mathrm{~min}$ time interval.

The one-day plot of the critical frequencies foF 2 and foE for 22 September 2016 is presented in Figure 2. The values of sounding frequencies 3.59 and $4.65 \mathrm{MHz}$ are also displayed (horizontal lines). DDM can be successfully performed when sounding frequency is under the critical frequency of the F2 layer $f o F 2$. It is evident that before 05 UT and after 20:20 UT the measurement could not be done at the frequency of $4.65 \mathrm{MHz}$. Frequency of $3.59 \mathrm{MHz}$ could be used for the measurements in the period between about 04 and about 21 UT. The critical frequency $f_{o} E$, including sporadic E layer (Es), is significantly below the sounding frequency of $3.65 \mathrm{MHz}$ during the entire day, so the measurement is not affected by any reflection from the $\mathrm{E}$ layer.

For this experiment, it is possible to compare directly the measured values of the Doppler shift by DDM and CDS at both sounding frequencies. The one-day CDS spectrograms are presented in Figure 3 at background with lighter colors. The upper left panel shows measurements for sounding frequency of $3.59 \mathrm{MHz}$ and the upper right panel shows measurements for sounding frequency of $4.65 \mathrm{MHz}$. Histograms from DDM are represented by brighter lines that are displayed at discrete times. Both the frequency and speed units are used in vertical axes in Figure 3 for better readability.
It is evident that signal on the CDS spectrogram is visible even if the critical frequency foF 2 of the F-layer drops below the sounding frequency. For frequency of $3.59 \mathrm{MHz}$ CDS produces continuous spectrogram practically during the whole investigated day. It is because CDS receives extraordinary wave in this case; the critical frequency for the extraordinary wave, $f x F 2$ is higher than for ordinary wave, $f x F 2 \sim f o F 2+$ $0.6 \mathrm{MHz}$.

For frequency of $4.65 \mathrm{MHz}$ we have CDS signal in the time between 05 and 21 UT. For DDM measurements, the Digisonde distinguishes the ordinary and extraordinary mode of signal. Only reflection points detected for an ordinary mode are processed. The CDS is not able to distinguish between ordinary and extraordinary mode and even if the critical frequency foF 2 drops below the sounding frequency, the extraordinary mode might be present in the CDS spectrogram. The main feature of the diurnal cycle is the same for both the CDS and DDM curves.

Lower panels of Figure 3 show detailed views. Two-hour time window of the spectrogram for sounding frequency of $3.59 \mathrm{MHz}$ is displayed on the left. The right panel shows the same time window for sounding frequency of $4.65 \mathrm{MHz}$. The detailed views show the time period between 16 and $18 \mathrm{UT}$. Significant oscillations with period about $20 \mathrm{~min}$ are visible in the CDS spectrograms for both sounding frequencies. The oscillations are detected also in DDM data. It is however clear that the detection and analysis of these shorter periods is significantly better/easier by the CDS method. It is necessary to emphasize that the Digisonde performed the drift measurements with much higher cadence during this experiment than it is usual for the normal operation.

The strength of CDS is demonstrated in this picture (lower panel of Fig. 3). The detection and analysis of short-period fluctuations is therefore possible using this method. CDS 



Fig. 3. Comparison of the CDS spectrograms (background) and DDM histograms (discrete vertical lines) for a special campaign (Sep 22, 2016). The upper panels show the one-day plots, lower panels show the detailed view for time window 16-18 UT. Left panels show result for sounding frequency of $3.59 \mathrm{MHz}$, right panels show result for sounding frequency of $4.65 \mathrm{MHz}$.

makes it possible to detect and analyze periods longer than about 12-15 s (Chum et al., 2016a). Analysis of periods shorter than about $30 \mathrm{~min}$ is practically impossible in standard routine DDM operation.

The combination of both methods can help us to get a very detailed and comprehensive view of the wave processes in the ionosphere.

\subsection{Basic types of spectrograms}

According to our experience, several basic spectrogram types occur. We investigate whether they correspond to special types of SKYmaps and/or ionograms. Different types of spectrograms and their comparison with histograms obtained from SKYmap are shown in the following examples. In this study, the actual sounding frequencies are different for each case of DDM and sounding frequency for DDM is usually not equal to the CDS sounding frequency.

Thin line. A single narrow peak in the CDS spectra is shown as a clear thin line in the spectrogram. It mostly relates to the cases when the DDM detects only a few reflection points near vertical direction. The DDM usually does not estimate horizontal components of the velocity vector with a sufficient precision in such cases (Kouba and Koucká Knížová, 2012). Only the estimated vertical component of drift velocity fulfills the required accuracy. Such situation often persists for hours. Data quality for estimated horizontal velocity components rapidly fluctuates or the data are missing. Therefore, it is difficult to analyze the horizontal components and credible publications on this topic are rare. The vertical velocity component is not affected by this problem. On the other hand, analysis of horizontal propagation of traveling ionospheric disturbances and gravity waves is possible for such cases provided that multi-point CDS is performed (Chum et al., 2014). The horizontal velocities and directions are determined from the time delays between the observations of corresponding fluctuations on different sounding paths.

The upper panel of Figure 4 shows the two-hour thin line type spectrogram. The DDM measurement is displayed in Figure 4 with saturated colors. The DDM was carried out in autodrift mode with 15 min interval. It is obvious that maxima 

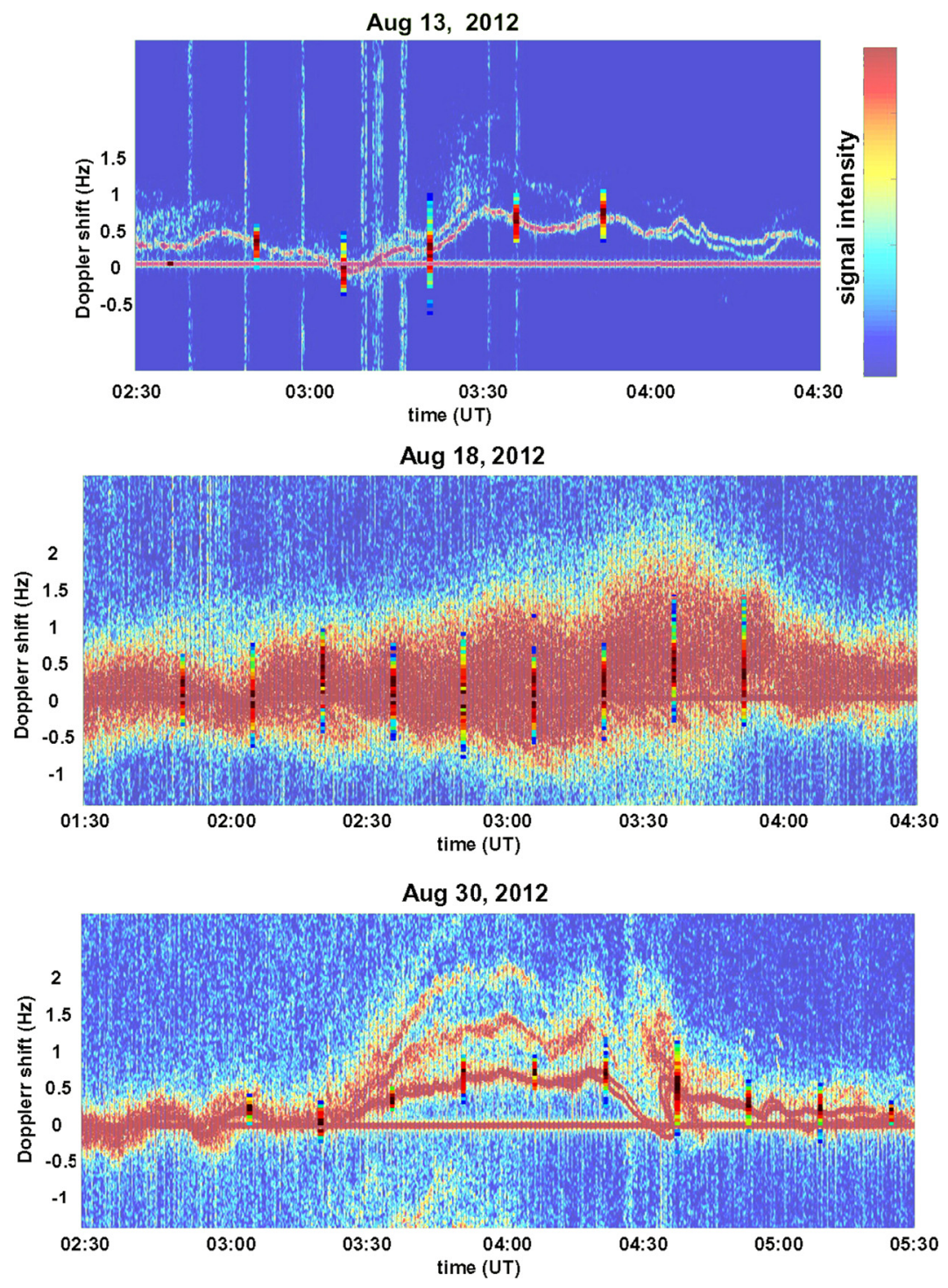

Fig. 4. Comparison of the CDS and the DDM for a different ionospheric conditions. The CDS spectrograms are on background and DDM histograms shown by discrete vertical lines. The upper panel: the spectrogram signal is a thin line. The middle panel: the spectrogram for spread F conditions. The lower panel: the spectrogram shows multiple hop reflections.

of detected DDM peaks correspond exactly to observed trace in the Doppler shift spectrograms obtained by the CDS.

Spread F. In contrast with previous case, sometimes a broad signal on a spectrogram appears. Such situations can be observed for several hours. Thick spectrogram corresponds to the Spread F in the ionogram. The Spread F condition is a typical summer phenomena in mid-latitudes. In such case, the DDM produces SKYmap with plenty of points distributed in a broad area, which means that the velocity vector can be estimated with a high accuracy. Such situations are ideal for precise DDM measurements.

Spread F can be significant disturbance of GNSS signal and decrease the precision of position determination.

The middle panel of Figure 4 shows three-hour spread type spectrogram and its comparison with the DDM results.

Multiple lines. In this case the thin line on the CDS spectrogram is split into several lines.
Multiple subsequent reflections from F-layer and ground are observed in this case. The lines appear one above another, as it shown in the lower panel of Figure 4. A comparison with the DDM shows that the line with the lowest Doppler shift corresponds to a single F layer reflection. The Doppler shifts of multiple reflections are higher, e.g., the double reflection is represented by the Doppler shift that is two-times larger than that for the single reflection.

The DDM can also detect multiple reflections but they are eliminated during data processing. Selection of points for correct velocity estimates was proposed by Kouba et al. (2008). Correct height range should be used for the velocity calculations.

It is clear that the requirement for using selected correct reflection points for velocity calculation in DDM is justified. It is evident that the signal for multiple reflections has a different Doppler value and would therefore distort the estimated 


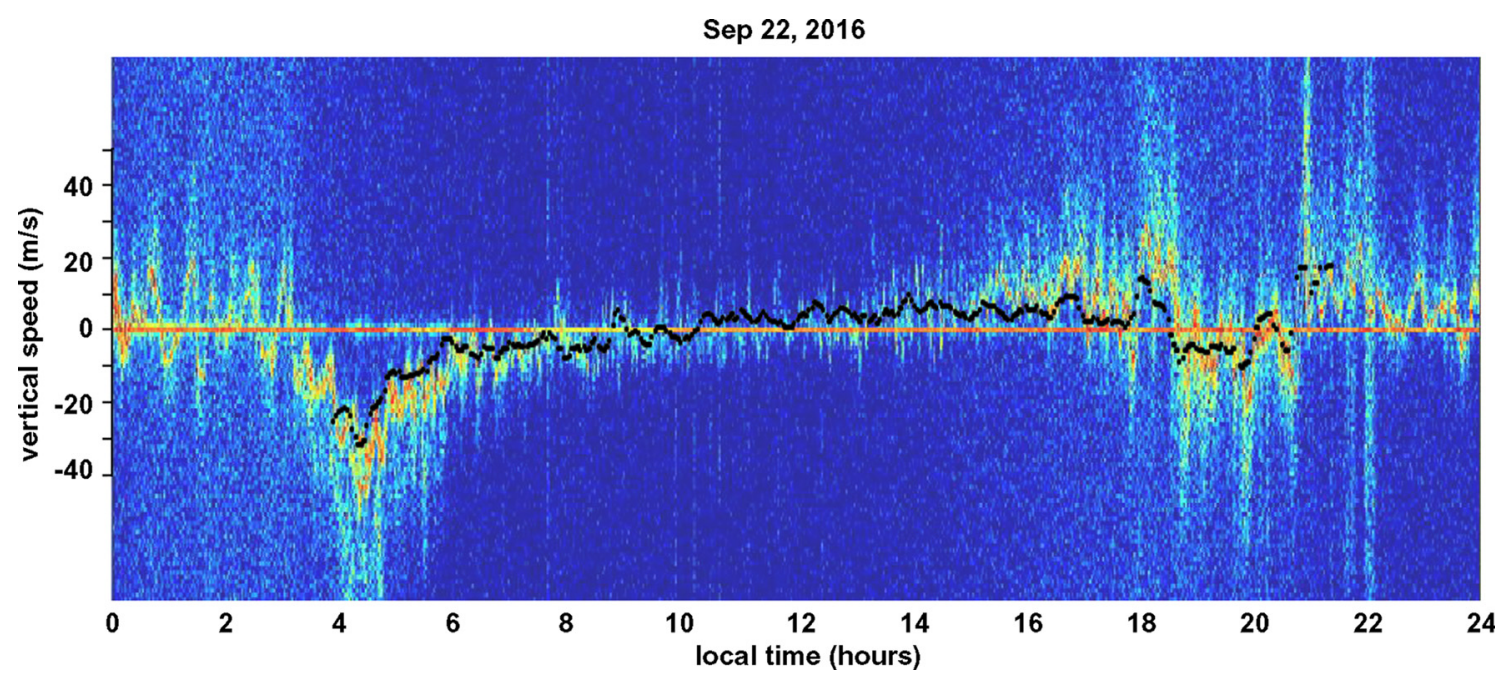

Fig. 5. Comparison of the vertical drift velocity component calculated using the DDM (black dots) and the CDS spectrogram for sounding frequency of $3.59 \mathrm{MHz}$ (spectrogram is plotted in speed units). Shown is the one-day plot for September 22, 2016.

velocity. Despite this fact, the automatic processing of reflection points is occasionally used and can affect the results (Mathew et al., 2017).

It should be noted that the multiple lines can also be observed in the Doppler shift spectrogram when the ordinary (L-O) and extraordinary (R-X) wave modes reflect in different regions and experience different Doppler shifts. However, the ratio of Doppler shifts for L-O and R-X wave modes is usually different from integer in such cases (splitting to L-O and R-X mode can be observed from $\sim 4: 00$ to $\sim 4: 25$ UT in the upper spectrogram).

\subsection{The CDS spectrogram vs. DDM vertical velocity}

We have compared the detected values of line-of-sight velocity for both methods.

In the DDM case, the line-of-sight velocity histograms were displayed. The CDS spectrograms were also set in the velocity units.

DDM uses the data to estimate the velocity components which is a final product of the drift measurement. Sometimes it is possible to determine all components of the velocity vector with good precision. However, it is possible to determine only a vertical component when only reflections from a nearvertical direction are detected.

The CDS path Pruhonice-Prague represents a vertical sounding of the ionosphere (the distance of transmitter and receiver is only about $7 \mathrm{~km}$ ). Therefore, the detected spectrograms can be often interpreted as the vertical component of the drift velocity. To prove this, we must compare CDS and DDM measurements in a specific time interval.

Figure 5 shows a comparison of CDS spectrogram at $3.59 \mathrm{MHz}$ with a vertical velocity component $v_{z}$ calculated by the DDM. There is one-day plot for September 22, 2016 in the figure. The spectrogram scaled in $\mathrm{m} / \mathrm{s}$ is on its background. Vertical velocity components estimated by DDM are presented as black points. The $30 \mathrm{~min}$ running mean was used for the DDM data. It is evident that a very good agreement was obtained in the most cases. Slightly higher CDS values are observed around sunrise. Thus, CDS for short path measures mostly the vertical velocity component and can be compared with the final DDM result.

This information is important because CDS produces data with excellent temporal resolution. For a detailed study of $v_{z}$ there is no need to perform time-consuming DDM measurements, but it is possible to use CDS with high time resolution. An exception might be cases when there are large differences between the shape of rising and falling edge of the Doppler shift fluctuations in the Doppler shift record or when the Sshaped patterns are observed in the Doppler shift spectrograms (Davies and Baker, 1966; Chum et al., 2012). In such cases, oblique reflections from horizontal propagating perturbations might contribute to the observed Doppler shifts (see also discussion in the Section Asymmetric SKYmap problem).

The data presented in Figure 5 were collected during special high-rate sampling campaign discussed in Section 3.1. The campaign was realized during quiet geomagnetic conditions.

The behavior of vertical velocity plot agrees with quiettime diurnal variation presented by Kouba and Koucká Knížová (2016). Dominant detected disturbanes in one-day plot are regular peaks related to sunrise/sunset. Amplitudes of other disturbances are significantly lower.

\subsection{Asymmetric SKYmap problem}

We mentioned minor differences in results near sunrise in the last paragraph. This can be explained as follows. We assumed that the main CDS signal will come from the vertical direction for the short path. In some situations, this assumption is not valid. When significant inhomogeneities occur, then the signal direction can divert from vertical. It is appropriate to analyze SKYmap to demonstrate such situation. Typically, there is an amount of reflection points with the center of the cloud in the "origin". Such case produces a very good agreement with the CDS. However, the cloud can be situated asymmetrically with respect to the origin. The main signal in CDS spectrogram follows the center of the cloud. Such 


\section{Pruhonice: Feb 05, 2017; 20:35 UT}

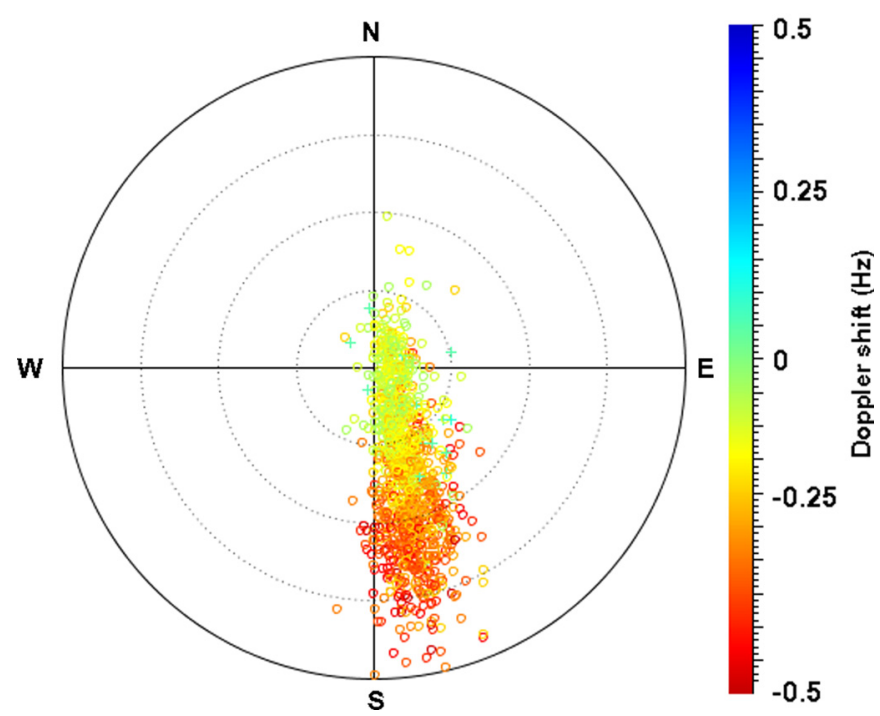

Fig. 6. Example of asymmetric SKYmap. The measurement was performed in Pruhonice on Feb 05, 2017 at 20:35 UT.

SKYmaps are occasionally recorded in Pruhonice but are not frequent.

An example of a significantly asymmetric SKYmap is in Figure 6. The SKYmap was recorded on February 5, 2017 at Pruhonice station. Altogether 1150 reflection points were detected during the measurements with sounding frequency window from 1.7 to $2.05 \mathrm{MHz}$.

The reflection points form a cloud. However, the reflection points corresponding to vertical direction are situated practically on the edge of the cloud. Most of the detected reflection points are in the south-east quadrant. A small number of reflections were detected in the north-east quadrant. Almost no reflection points were detected in the west direction. The detected reflection points cover sufficiently large area and it is no problem to estimate full velocity vector with good accuracy. The accuracy of the individual components will depend on the coverage of reflection points in the specific direction. The north-south component can be determined with very good accuracy. The center of the reflection points cloud is deflected about 15-20 degrees from the vertical to the SSE direction. That means that the center of the signal recorded by the CDS will correspond to that direction. Consequently, it is not possible to interpret the CDS as vertical sounding in this case. Occurrences of such problematic measurements can be detected by a common evaluation of DDM and CDS data.

Possible differences are expected when distinct short-scale disturbances or the solar terminator pass over the sounded volume. Nevertheless, the sounding can be considered as vertical in most cases.

\section{Conclusion}

The paper compares the possibilities of the CDS and the DDM methods for research and monitoring of ionospheric dynamics. A special campaign was performed to compare the results of both methods on the basis of identification of various typical phenomena that occur in the ionosphere as TIDs, spread F, and significant and small horizontal electron density gradients. The results were consistent and are in mutual agreement. The main advantage of the CDS method is its excellent temporal resolution. The main advantage of the DDM is the detailed information on the detected reflection points. The CDS spectrogram can usually be interpreted as the vertical component of the drift velocity, provided that the transmitter and receiver are sufficiently close. The CDS can be used as a tool to identify presence of characteristic wave activity, particularly TID, by means of computation of vertical velocity component. In such case, the DDM can serve as a complementary method to provide detailed information about the state of the ionosphere during the studied event. Further, combination of multiple CDS paths can be used for description of horizontal wave propagation computed from phase shift between the detected structures on the spectrograms.

We conclude that both methods are complementary and that their simultaneous operation is advantageous for detailed description of the ionospheric dynamics.

Acknowledgement. DK would like to thank to the project 15-24688S supported by the Czech Science Foundation and JC acknowledges the support under the grant $15-07281 \mathrm{~J}$ by the Czech Science Foundation. The editor thanks two anonymous referees for their assistance in evaluating this paper.

\section{References}

Banks PM, Rino CL, Wickwar VB. 1974. Incoherent scatter radar observations of westward electric fields and plasma densities in the auroral ionosphere. J Geophys Res 79: 187-198.

Bertoni F, Batista IS, Abdu MA, Reinisch BW, Kherani EA. 2006. A comparison of ionospheric vertical drift velocities measured by Digisonde and Incoherent Scatter Radar at the magnetic equator. $J$ Atm Solar-Terr Phys 68: 669-678.

Chou MY, Lin CCH, Yue J, Tsai HF, Sun YY, Liu JY, Chen CH. 2017. Concentric traveling ionosphere disturbances triggered by Super Typhoon Meranti. Geophys Res Lett 44: 1219-1226, DOI: 10.1002/ 2016 GL072205.

Chum J, Athieno R, Baše $J$, Burešová D, Hruška F, Laštovička $J$, McKinnell LA, Šindelářová T. 2012. Statistical investigation of horizontal propagation of gravity waves in the ionosphere over Europe and South Africa. J Geophys Res 117: A03312, DOI: 10.1029/2011JA017161.

Chum J, Bonomi FAM, Fišer J, Cabrera MA, Ezquer RG, et al. 2014. Propagation of gravity waves and spread $\mathrm{F}$ in the low-latitude ionosphere over Tucumán, Argentina, by continuous Doppler sounding: First results. J Geophys Res Space Phys 119: 6954-6965, DOI: 10.1002/2014JA020184.

Chum J, Liu YJ, Laštovička J, Fišer J, Mošna Z, Baše J, Sun YY. 2016a. Ionospheric signatures of the April 25, 2015 Nepal earthquake and the relative role of compression and advection for Doppler sounding of infrasound in the ionosphere. Earth Planets Space 68: 24, DOI: 10.1186/s40623-016-0401-9.

Chum J, Liu YJ, Chen SP, Cabrera MA, Laštovička J, Baše J, Burešová D, Fišer J, Hruška F, Ezquer RG. 2016b. Spread F occurrence and drift under the crest of the equatorial ionization anomaly from continuous Doppler sounding and FORMOSAT-3/ COSMIC scintillation data. Earth Planets Space 68: 56, DOI: 10.1186/s40623-016-0433-1.

Davies K, Baker DM. 1966. On frequency variations of ionospherically propagated HF radio signals. Radio Sci 1: 545-556. 
Davies K, Watts J, Zacharisen D. 1962. A study of F2-layer effects as observed with a Doppler technique. J Geophys Res 67: 2, DOI: 10.1029/JZ067i002p00601.

Fejer BG, Paula ED, Gonzalez SA, Woodman RF. 1991. Average vertical and zonal $\mathrm{F}$ region plasma drifts over Jicamarca. J Geophys Res Space Phys 96: 13901-13906.

Fišer J, Chum J, Liu JY. 2017. Medium-scale traveling ionospheric disturbances over Taiwan observed with HF Doppler sounding. Earth Planets Space 69: 131, DOI: 10.1186/s40623-017-0719-y.

Georges TM. 1973. Infrasound from convective storms: examining the evidence. Rev Geophys Space Phys 11: 571-594.

Hajj GA, Ibanez-Meier R, Kursinski ER, Romans LJ. 1994. Imaging the ionosphere with the Global Positioning System. Int J Imaging Syst Technol 5: 174-187.

Jakowski N, Wilken V, Schlueter S, Stankov SM, Heise S. 2005. Ionospheric space weather effects monitored by simultaneous ground and space based GNSS signals. J Atm Solar-Terr Phys 67: 1074-1084.

Kouba D, Koucká Knížová P. 2012. Analysis of digisonde drift measurements quality. J Atm Solar-Terr Phys 90-91: 212-221.

Kouba D, Koucká Knížová P. 2016. Ionospheric vertical drift response at a mid-latitude station. Adv Space Res 58: 108-116.

Kouba D, Boška J, Galkin IA, Santolík O, Šauli P. 2008. Ionospheric drift measurements: Skymap points selection. Radio Sci 43: 1, RS1S90/1-RS1S90/11.

Laštovička J, Chum J. 2017. A review of results of the international ionospheric Doppler sounder network. Adv Space Res 60: 16291643.
Lay EH, Shao XM, Kendrick AK, Carrano CS. 2015. Ionospheric acoustic and gravity waves associated with midlatitude thunderstorms. J Geophys Res Space Phys 120: 6010-6020, DOI: 10.1002/ 2015JA021334.

Mathew TJ, Haralambous H, Oikonomou Ch. 2017. Pre-sunrise uplift and sunrise downward excursion in the F-region vertical plasma drift: observations from the mid-latitude station Nicosia. Adv Space Res 59: 1792-1799.

Nishioka M, Tsugawa T, Kubota M, Ishii M. 2013. Concentric waves and short-period oscillations observed in the ionosphere after the 2013 Moore EF5 tornado. Geophys Res Lett 40: 5581-5586, DOI: 10.1002/2013GL057963.

Otsuka Y, Suzuki K, Nakagawa S, Nishioka M, Shiokawa K, Tsugawa T. 2013. GPS observations of medium-scale traveling ionospheric disturbances over Europe. Ann Geophys 31: 163-172, DOI: 10.5194/angeo-31-163-2013.

Reinisch BW, Scali JL, Haines DM. 1998. Ionospheric drift measurements with ionosondes. Ann Geofis 41: 695-700.

Saito A, Fukao S, Miyazaki S. 1998. High resolution mapping of TEC perturbations with the GSI GPS Network over Japan. Geophys Res Lett 25: 3079-3082.

Scali JL, Reinisch BW, Heinselman CJ, Bullett T. 1995. Coordinated digisonde and incoherent scatter radar $\mathrm{F}$ region drift measurements at Sondre Stromfjord. Radio Sci 30: 1481-1498.

Tarantola A. 2005. Inverse Problem Theory and Methods for Model Parameter Estimation, Society for Industrial and Applied Mathematics, Philadelphia, PA, USA, ISBN: 0-89871-572-5. 